\title{
Fracturas diafisarias radiocubitales en pacientes pediátricos. Revisión de resultados de tratamiento
}

\author{
Reyes-Hernández LA, ${ }^{*}$ Cervantes-Gudiño JE, ${ }^{* *}$ García-Diosdado A*** \\ Centenario Hospital Miguel Hidalgo
}

RESUMEN. Objetivo: Describir los tipos de fracturas más comunes en pacientes menores de 16 años de edad a nivel de antebrazo y evaluar, dependiendo del nivel de la fractura, el tratamiento con mejores resultados. Material y métodos: Realizamos un análisis retrospectivo para determinar frecuencia de las fracturas de antebrazo en menores de 16 años, en el período de Enero 2013-Julio 2017. Se ingresó en sistema los siguientes diagnósticos: fractura de antebrazo, fractura de radio, fractura de cúbito y fractura radiocubital. Resultados: Se incluyeron 573 pacientes: $\mathbf{4 1 3}$ hombres, 160 mujeres; lado afectado derecho: 230, izquierdo 338, bilateral 5; nivel afectado: 438 distales, 102 medias y 33 proximales. La edad pico de presentación fue a los ocho años. El tratamiento inicial fue conservador en $92 \%$, seguido de reducción cerrada y fijación percutánea con $4.71 \%$ y se tuvo $2.4 \%$ para reducción abierta y fijación interna con placas. Se tuvo un tratamiento inicial fallido en $3.83 \%$ al encontrarse una reducción inadecuada. Discusión: Encontramos que el tratamiento de las fracturas en nuestra institución es conservador en $\mathbf{9 2 \%}$, se usa la reducción cerrada y fijación percutánea sólo en $\mathbf{4 . 7 1 \%}$ y sólo se realiza de primera intención la reducción abierta y fijación interna en $\mathbf{2 . 4 \%}$ siendo la mayor prevalencia en pacientes mayores de 12 años con fracturas diafisarias medias radiocubitales.

Palabras clave: Antebrazo, fracturas, tratamiento, niños, resultados.
ABSTRACT. Objective: To describe the most common types of fractures in patients under 16 years of age at the forearm level, and to evaluate depending on the level of the fracture, the treatment with better results. Material and methods: We perform a retrospective analysis to determine the frequency of forearm fractures in patients under 16 years, in the period from January 2013-July 2017. The following diagnoses were entered into the system: forearm fracture, radius fracture, ulnar fracture and bilateral fracture. Results: We included 573 patients: 413 male, 160 females; right affected side: 230, left: 338 , bilateral 5 . Affected level: 438 distal, 102 mid-shaft and 33 proximal. The peak age of presentation was eight years. The initial treatment was conservative in $92 \%$, followed by closed reduction and percutaneous fixation with 4.71 and $2.4 \%$ for open reduction and internal fixation with plates. There was a failed initial treatment at $3.83 \%$ when an inadequate reduction was found. Discussion: We found that the treatment of this fractures in our institution is conservative in $92 \%$, closed reduction and percutaneous fixation in $4.71 \%$ and open reduction and internal fixation in a $2.4 \%$ being The highest prevalence in patients older than 12 years with bilateral mid-shaft fractures.

Key words: Forearm, fractures, treatment, children, results.

\footnotetext{
* Médico Residente de cuarto año de la Especialidad de Traumatología y Ortopedia.

** Médico adscrito del Servicio de Traumatología y Ortopedia.

*** Médico interno de Pregrado.

Centenario Hospital «Miguel Hidalgo».
}

Dirección para correspondencia:

Dra. Lydia Alessandra Reyes-Hernández

Av. Gómez Morín S/N, La Estación Alameda, CP 20259, Aguascalientes, AGS. Tel: 4499946720

E-mail: 1ydia_reyes88@hotmail.com

Este artículo puede ser consultado en versión completa en http://www.medigraphic.com/actaortopedica 


\section{Introducción}

Las fracturas diafisarias de radio y cúbito son la tercera fractura más frecuente en la población pediátrica (Figura 1) y representan $13-40 \%$ del total de las fracturas. ${ }^{1,2}$ Históricamente, la mayoría de estas fracturas han sido tratadas de manera no quirúrgica, con reducción cerrada y esperando su consolidación. Recientemente ha habido una tendencia hacia un mayor manejo quirúrgico de estas fracturas, en un esfuerzo por mejorar los resultados. ${ }^{3}$

El tratamiento de estas fracturas depende de la edad, el tipo de desplazamiento de la fractura, entre otras. Teniendo en cuenta el potencial de crecimiento fisiario de un niño, un rango de grados de angulación se puede aceptar en función de la edad del niño y su capacidad de remodelación. ${ }^{4}$

La inmovilización del antebrazo con férulas o yesos circulares sigue siendo una opción de tratamiento viable para muchas de estas fracturas que están dentro de los parámetros aceptables de alineación (Figura 2). Generalmente existe bajo riesgo de desarrollar rigidez en el codo tras el tratamiento. Para trazos de fractura en los que los parámetros de alineación no permiten limitarse al tratamiento con reducción cerrada se recomienda el tratamiento quirúrgico. Las opciones de tratamiento quirúrgico incluyen la fijación con placas y con clavos intramedulares, que pueden ser rígidos o elásticos. Recientemente ha habido un interés creciente en la determinación de qué método proporciona resultados superiores, pero el tratamiento óptimo permanece controversial. ${ }^{5,6,7}$ Nuestro objetivo de trabajo fue describir los
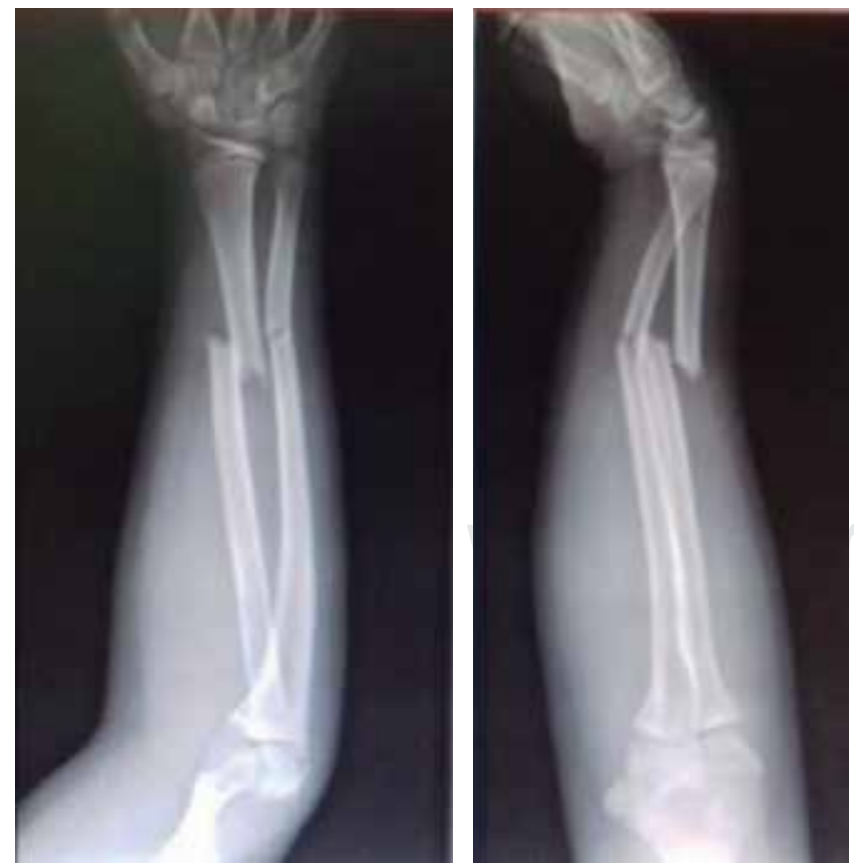

Figura 1: Radiografía anteroposterior y lateral de antebrazo. Fractura diafisaria distal. tipos de fracturas más comunes en pacientes menores de 16 años de edad a nivel de antebrazo y evaluar, dependiendo del nivel de la fractura, el tratamiento con mejores resultados, así como las complicaciones más frecuentes que se presentan.

\section{Material y métodos}

Realizamos un análisis retrospectivo para determinar frecuencia de las fracturas de antebrazo en menores de 16 años, en el período de Enero 2013-Julio 2017. Se ingresó en nuestro sistema de expedientes los siguientes diagnósticos: fractura de antebrazo, fractura de radio, fractura de cúbito y fractura radiocubital. Como criterios de inclusión se tomó en cuenta que el expediente electrónico y radiográfico estuviera completo. Los datos recuperados incluyeron edad del paciente, sexo, lado afectado, tratamiento inicial y necesidad de otro tratamiento posterior, así como estado actual del sujeto. Los casos excluidos fueron aquellos que tenían expediente o historial radiográfico incompleto, altas voluntarias, pacientes mayores de 16 años y casos con diagnóstico de contusión o esguince.

\section{Resultados}

Se obtuvo en total 2,794 expedientes con la búsqueda inicial (fractura de antebrazo, fractura de radio, fractura de cúbito y fractura radiocubital). Descartando 729 por tener un expediente incompleto y 1,143 por ser mayores de 16 años.

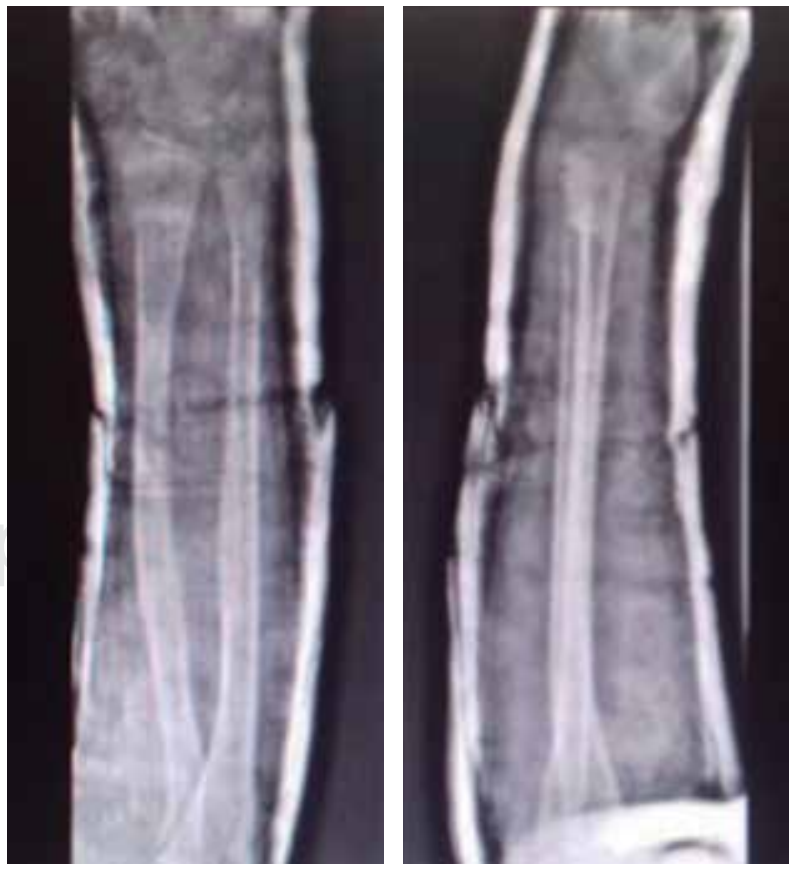

Figura 2: Control post-reducción cerrada e inmovilización con yeso circular. 


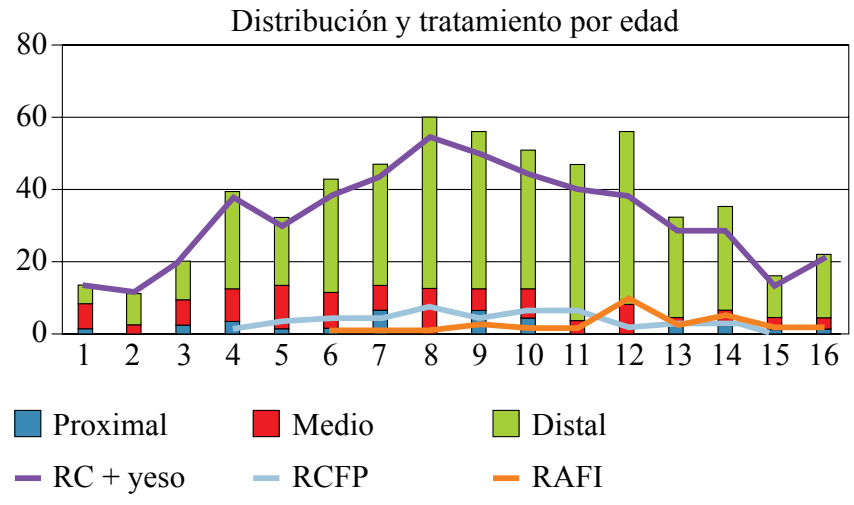

$\mathrm{RC}=$ reducción cerrada; $\mathrm{RCFP}=$ reducción cerrada y fijación percutánea; $\mathrm{RAFI}=$ reducción abierta y fijación interna.

Figura 3: Distribución de tratamiento de acuerdo al sitio de fractura y tratamiento inicial.

Teniendo un total de 922 pacientes menores de 16 años, de los cuales 352 fueron eliminados del estudio por contar con diagnóstico de contusión o esguince y seis por egresarse voluntariamente y no contar con seguimiento del padecimiento.

Se estudiaron 573 pacientes con diagnóstico de fractura, obteniendo los siguientes resultados: 413 hombres, $160 \mathrm{mu}-$ jeres; lado afectado derecho 230, izquierdo 338 , bilateral 5; nivel afectado: 438 distales, 102 medias y 33 proximales. El radio fue el hueso principalmente afectado con $61 \%$, seguido por las fracturas radiocubitales con $35 \%$ y el cúbito con $4 \%$. La edad pico de presentación fue a los ocho años.

El tratamiento inicial fue conservador en $92 \%$, seguido de reducción cerrada y fijación percutánea con $4.71 \%$ y se tuvo $2.4 \%$ para reducción abierta y fijación interna con placas (Figura 3).

El tratamiento inicial por localización de fractura: de los 33 pacientes con fracturas proximales, 29 tratados con reducción cerrada y yeso braquipalmar, otros tres con reducción cerrada con clavillos Kirschner y el paciente restante con reducción abierta con placas. A nivel diafisario medio con un total de 101, 76 fueron tratados con yeso braquipalmar, 12 con reducción cerrada y fijación percutánea con clavillos; 13 con reducción abierta con placas, teniendo una mayor incidencia a partir de los 12 años de edad por contar con una inestabilidad al estar ambos huesos fracturados al mismo nivel. Y de las diafisarias distales 410: reducción cerrada y colocación de yeso braquipalmar en 396, 13 reducción cerrada y fijación percutánea, reducción abierta y fijación interna en dos.

Se tuvo un tratamiento inicial fallido en $3.83 \%$ al encontrarse una reducción inadecuada; se retiró el yeso braquipalmar y se realizó reducción cerrada y fijación percutánea en 15 pacientes (seis con fractura diafisaria media y nueve con fractura diafisaria distal). En seis casos se retiró el yeso braquipalmar y se realizó reducción abierta y fijación interna con placas (cuatro con fractura diafisaria media y dos con fractura distal) y sólo se tuvo que realizar el cambio en un paciente con reducción cerrada y fijación percutánea a reducción abierta y fijación interna con placa por presentar retardo en la consolidación.

\section{Discusión}

Las fracturas del antebrazo son lesiones comunes en los niños pues representan $62 \%$ de las fracturas de las extremidades superiores. ${ }^{8,9,10} \mathrm{En}$ el análisis encontramos que se tiene un porcentaje en cuanto a distribución de sitio de fractura similar a lo reportado en la literatura mundial, teniendo $76.4 \%$ de las fracturas del antebrazo en el tercio distal, $17 \%$ en el tercio medio y $5.75 \%$ en el tercio proximal del antebrazo. . $^{11,12,13,14}$

Encontramos que el tratamiento de las fracturas en nuestra institución es conservador en $92 \%$, se usa la reducción cerrada y fijación percutánea sólo en $4.71 \%$ y sólo se realiza de primer intención la reducción abierta y fijación interna en $2.4 \%$ siendo la mayor prevalencia en pacientes mayores de 12 años con fracturas diafisarias medias de ambos huesos. A diferencia de la literatura mundial que reporta una tasa de complicaciones de hasta de $32 \%,{ }^{15,16}$ en nuestra institución se tuvo en el período estudiado sólo $3.83 \%$ de necesidad de un segundo tratamiento por no encontrarse una adecuada reducción, siendo los pacientes de ocho años los que presentan $0.7 \%$ de necesidad de un segundo tratamiento por no encontrarse una reducción aceptable. Debemos estar familiarizados con todas las opciones de tratamiento. Es importante abordar cada fractura como única y no tratarlas todas con una sola técnica. El conocimiento de las indicaciones, resultados y complicaciones potenciales de estos métodos es un requisito para el óptimo tratamiento de las fracturas.

\section{Bibliografía}

1. Cheng JC, Ng BK, Ying SY, Lam PK. A 10-year study of the changes in the pattern and treatment of 6,493 fractures. J Pediatr Orthop. 1999; 19(3): 344-50.

2. Jones K, Weiner DS. The management of forearm fractures in children: a plea for conservatism. J Pediatr Orthop. 1999; 19(6): 811-5.

3. Flynn JM, Jones KJ, Garner MR, Goebel J. Eleven years experience in the operative management of pediatric forearm fractures. J Pediatr Orthop. 2010; 30(4): 313-9.

4. Mahecha-Toro M, Vergara-Amador E, González Ramírez M. Fracturas diafisarias de antebrazo en niños: tratamiento con fijación intramedular con clavos Kirschner. Rev Esp Cir Ortop Traumatol. 2018; 62(1): 71-79.

5. Abraham A, Kumar S, Chaudhry S, Ibrahim T. Surgical interventions for diaphyseal fractures of the radius and ulna in children. Cochrane Database Syst Rev. 2011; (11): CD007907.

6. Tachdjian MO. Ortopedia pediátrica. Madrid: Marbán; 2003, Tomo IV, 3419-21.

7. Sinikumpu JJ, Pokka T, Serlo W. The changing pattern of pediatric both-bone forearm shaft fractures among 86,000 children from 1997 to 2009. Eur J Pediatr Surg. 2013; 23(4): 289-96.

8. Rennie L, Court-Brown CM, Mok JY, Beattie TF. The epidemiology of fractures in children. Injury. 2007; 38(8): 913-22.

9. Ryan LM, Teach SJ, Searcy K, Singer SA, Wood R, Wright JL, et al. Epidemiology of pediatric forearm fractures in Washington, DC. $J$ Trauma. 2010; 69(4 Suppl): S200-5. 
10. Rivara FP, Grossman DC, Cummings P. Injury prevention. First of two parts. N Engl J Med. 1997; 337(8): 543-8.

11. Rodríguez-Merchán EC. Pediatric fractures of the forearm. Clin Orthop Relat Res. 2005; (432): 65-72.

12. Canale ST, Beaty JH. Forearm Fractures. In: Campbell WC, Canale ST, Beaty JH. Campbell's operative orthopaedics. 11th ed. Philadelphia, PA: Mosby/Elsevier; 2008.

13. Noonan KJ, Price CT. Forearm and distal radius fractures in children. J Am Acad Orthop Surg. 1998; 6(3): 146-56.
14. Jouve JL. Fracturas del antebrazo en el niño. Aparato locomotor. EMC. 1997: 14-045-A-10.

15. Lobo-Escolar A, Roche A, Bregante J, Gil-Alvaroba J, Sola A, Herrera A. Delayed union in pediatric forearm fractures. J Pediatr Orthop. 2012; 32(1): 54-7.

16. Sinikumpu JJ, Lautamo A, Pokka T, Serlo W. Complications and radiographic outcome of children's both-bone diaphyseal forearm fractures after invasive and non-invasive treatment. Injury. 2013; 44(4): 431-6. 